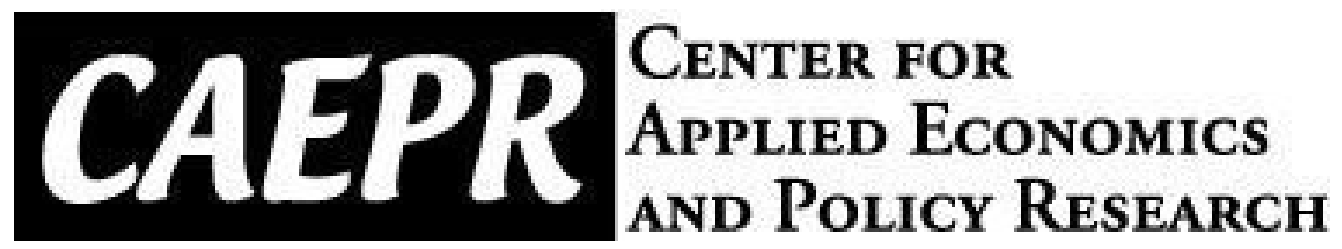

\author{
CAEPR \\ Working Paper \\ \#2017-014
}

\title{
Identification and Generalized Band Spectrum Estimation of the New Keynesian Phillips Curve
}

\author{
Jinho Choi \\ AMRO and Bank of \\ Korea \\ Juan Carlos \\ Escanciano \\ Indiana University \\ Junjie Guo \\ Indiana University
}

November 22, 2017

This paper can be downloaded without charge from the Social Science Research Network electronic library at https://papers.ssrn.com/abstract_id=3078201

The Center for Applied Economics and Policy Research resides in the Department of Economics at Indiana University Bloomington. CAEPR can be found on the Internet at: http://www.indiana.edu/ caepr. CAEPR can be reached via email at caepr@indiana.edu or via phone at 812-855-4050.

(C)2017 by Jinho Choi, Juan Carlos Escanciano and Junjie Guo. All rights reserved. Short sections of text, not to exceed two paragraphs, may be quoted without explicit permission provided that full credit, including (C) notice, is given to the source. 


\title{
Identification and Generalized Band Spectrum Estimation of the New Keynesian Phillips Curve
}

\author{
Jinho Choi* \\ AMRO and Bank of Korea \\ Juan Carlos Escanciano ${ }^{\dagger}$ \\ Indiana University \\ Junjie Guo \\ Indiana University
}

November 22nd, 2017.

\begin{abstract}
This article proposes a new identification strategy and a new estimation method for the hybrid New Keynesian Phillips curve (NKPC). Unlike the predominant Generalized Method of Moments (GMM) approach, which leads to weak identification of the NKPC with U.S. postwar data, our nonparametric method exploits nonlinear variation in inflation dynamics and provides supporting evidence of point-identification. This article shows that identification of the NKPC is characterized by two conditional moment restrictions. This insight leads to a quantitative method to assess identification in the NKPC. For estimation, the article proposes a closed-form Generalized Band Spectrum Estimator (GBSE) that effectively uses information from the conditional moments, accounts for nonlinear variation, and permits a focus on short-run dynamics. Applying the GBSE to U.S postwar data, we find a significant coefficient of marginal cost and that the forward-looking component and the inflation inertia are both equally quantitatively important in explaining the short-run inflation dynamics, substantially reducing sampling uncertainty relative to existing GMM estimates.
\end{abstract}

JEL Classification: C26, C51; E31; E37

Keywords: Point-identification; New Keynesian Phillips curve; Weak instruments; Nonlinear dependence; Generalized spectrum.

*Email address: choi.jinho@amro-asia.org. The findings, interpretations, and conclusions expressed in this material represent the views of the author and are not necessarily those of the ASEAN +3 Macroeconomic Research Office (AMRO) or its member authorities. Neither AMRO nor its member authorities shall be held responsible for any consequence of the use of the information contained herein.

${ }^{\dagger}$ Department of Economics, 105 Wylie Hall, 100 S. Woodlawn, Bloomington, IN 47405, U.S.A.; E-mail address: jescanci@indiana.edu. This research was funded by Spanish Plan Nacional de I+D+I grant numbers SEJ2007-62908 and ECO2014-55858-P.

${ }^{\ddagger}$ Ph.D Candidate, Department of Economics, 105 Wylie Hall, 100 S. Woodlawn, Bloomington, IN 47405, U.S.A.; E-mail address: junjguo@umail.iu.edu. 


\section{Introduction}

Economists have long been interested in studying the relationship between rates of inflation and unemployment (or related measures of real economic activity), see Phillips (1958). Inspired by a sticky pricing framework of Calvo (1983) and the inability of pure forward looking models to explain inflation dynamics, Galí and Gertler (1999) proposed a hybrid New Keynesian Phillips curve (NKPC), relating the current inflation to labor income shares, as a proxy for real marginal costs, and past and expected future inflations. Since its inception in the 1980s and 1990s, the NKPC has grown in popularity to become the key price determination equation in policy models used at central banks around the globe. Much of the NKPC's gained popularity comes from its solid theoretical microfoundations and what appeared to be an early empirical success of limited-information estimation methods, such as the Generalized Method of Moments (GMM) of Hansen (1982). See the seminal papers by Roberts (1995), Fuhrer and Moore (1995), Galí and Gertler (1999), and Sbordone (2002).

The early empirical success of GMM estimation of the NKPC was followed by an extensive literature pointing out to problems of weak identification and to the high sensitivity of these estimates to different specifications and instruments; see Mavroeidis, Plagborg-Møller, and Stock (2014) for a comprehensive survey of this literature, and Ma (2002), Mavroeidis (2005), Dufour, Khalaf, and Kichian (2006), Nason and Smith (2008a) and Kleibergen and Mavroeidis (2009) for early contributions along this line. The weak identification of the NKPC has motivated the development of identification-robust methods for testing and confidence intervals, but these methods are still based on the rather limited information provided by linear correlations between the instruments chosen and rates of inflation. ${ }^{1}$ The overall consensus is that this literature has reached a limit on what can be learned about the NKPC from standard identification strategies based on GMM, and that new identification approaches are needed.

This article proposes a new identification and estimation strategies that combine two novel features within the context of the NKPC: (i) a method that exploits nonparametrically nonlinear dependence in inflation dynamics, effectively using more information from the conditional moments than traditional GMM methods; and (ii) a method that allows researchers to focus on short run dynamics, where the strength of the relation between inflation and real economic activity is stronger. The methodological contributions translate into more stable and reliable inferences on the NKPC with postwar U.S data, and have applications beyond the NKPC.

\footnotetext{
${ }^{1}$ Because inflation is notoriously hard to forecast by linear methods once we control for lagged inflation, not much information is available from commonly used unconditional moments. As a result, weak-identificationrobust confidence sets for NKPC parameters often cover a substantial part of the parameter space and are not very informative (cf. Mavroeidis, Plagborg-Møller, and Stock (2014)).
} 
To illustrate the identification strategy of this article, let us consider first a toy example of a single-equation Rational Expectation (RE) model satisfying

$$
y_{t}=\gamma y_{t-1}+\alpha E\left[z_{t+1} \mid I_{t}\right]+v_{t}
$$

where $z_{t}$ can be equal to $y_{t}, v_{t}$ is an unpredictable error term, i.e. $E\left[v_{t} \mid I_{t-1}\right]=0$, and $I_{t}$ is the agent's information at time $t$. The standard approach in the RE literature replaces $E\left[z_{t+1} \mid I_{t}\right]$ by $z_{t+1}$, which yields a linear regression

$$
y_{t}=\gamma y_{t-1}+\alpha z_{t+1}+u_{t}
$$

where $u_{t}:=\alpha E\left[z_{t+1} \mid I_{t}\right]-\alpha z_{t+1}+v_{t}$. The regressor $z_{t+1}$ becomes endogenous in (2), rendering least squares estimates inconsistent, but the parameters $\boldsymbol{\theta}=(\gamma, \alpha)^{\prime}$ can be estimated from (2) by GMM, provided a valid instrument for $z_{t+1}$, different from $y_{t-1}$, is available.

The approach of this article is different. Define the prediction error $\varepsilon_{t}:=y_{t}-E\left[y_{t} \mid I_{t-1}\right]$ and note that (1) implies by the law of iterated expectations,

$$
y_{t}=\gamma y_{t-1}+\alpha E\left[z_{t+1} \mid I_{t-1}\right]+\varepsilon_{t}
$$

Instead of deriving inference from equation (2), we substitute the "first-stage" regression

$$
E\left[z_{t+1} \mid I_{t-1}\right]=\delta y_{t-1}+n_{t-1},
$$

in the equation (3), and obtain the reduced form equation

$$
y_{t}=(\gamma+\delta \alpha) y_{t-1}+\alpha n_{t-1}+\varepsilon_{t}
$$

Identification of the reduced form parameters, $\delta,(\gamma+\delta \alpha)$ and $\alpha$, imply identification of the structural parameters $\gamma$ and $\alpha$, provided the "nonlinearity in $y_{t-1}$ term" $n_{t-1}$, is nonzero. This is a classical identification argument. What appears to be new here is the use of (nonparametric) nonlinearity as an instrument and the focus on identification from the conditional moment rather than from unconditional ones as in GMM. Note that identification might be possible even when moments such as $E\left[z_{t+1} y_{t-2}\right]$ are close to zero (i.e. when $y_{t-2}$ is a weak instrument).

This article builds on these arguments and shows that identification in these models can be quantified by means of conditional moment restrictions. This is straightforward in the model above with one endogenous variable, but a similar characterization becomes more challenging when two endogenous variables are present, as is potentially the case for the 
NKPC. We characterize identification in the NKPC by two conditional moment restrictions, which is the basis for our quantitative identification analysis of the NKPC. The proposed identification tests can be interpreted as nonparametric analogues of the commonly used first stage $F$-tests in linear regression (see Stock and Yogo (2005)).

Despite the positive identification result above, a practical estimation method that incorporates this identification strategy seems difficult because nonparametric estimation of $E\left[z_{t+1} \mid I_{t-1}\right]$ is not feasible when $I_{t-1}$ is high-dimensional and sample sizes are moderate. We show how to overcome this difficulty by means of a generalized spectral approach based on a continuum of unconditional moments. This estimation method extends the estimator of Dominguez and Lobato (2004) and Escanciano (2017) to a spectral setting with an infinite number of lags, and it is of independent interest.

In this article these ideas are applied to the hybrid NKPC. This is a particularly relevant application of our methods because GMM has been shown to deliver weak identification and unstable inference. The first contribution of this article is the characterization of the nonparametric identification of the NKPC in terms of testable conditional moment restrictions, which extends the identification argument in the example above to the NKPC setting. We empirically test these restrictions with U.S. postwar data and find that nonlinearities in inflation dynamics are quantitatively important so as to suggest strong point-identification of the NKPC with U.S. postwar data, in contrast to the well-documented GMM methods that only deliver weak identification. We also show that endogenous regime switching models imply theoretically the kind of nonlinearity needed for our identification arguments.

The second contribution of this article is to propose a novel estimation method for linear conditional moment restrictions that accounts for nonlinear variation and allows researchers to focus on a particular band of frequencies, e.g. short run dynamics in the hybrid NKPC. This new estimator, which we term the Generalized Band Spectrum Estimator (GBSE), has a closed-form expression, and therefore, it is straightforward to implement. Applying the GBSE to U.S. postwar data reveals a significant coefficient of marginal cost and that the forward-looking component and the inflation inertia are both quantitatively important and statistically significant in explaining the U.S. short-run inflation dynamics. These empirical findings survive to different robustness checks, thereby showing that inferences for the NKPC based on the GBSE are more stable than those based on GMM and related methods.

Our estimates have important implications for policy (see Schmitt-Grohé and Uribe (2008)). The larger values obtained for the coefficient of marginal cost with the GBSE suggests a more important role for monetary policy than for related GMM methods. Our estimates imply that the Philips curve remains alive, which lend some support to central banks - especially inflation targeters - that may assess and stabilize the output gap in the 
short run with a view to achieving the medium-term inflation objectives, based on the Philips curve relationship. Our estimate for the coefficient of expected future inflation suggests a significant role for expectations anchoring and communications as tools of monetary policy, but with a smaller magnitude than previous GMM estimates. Finally, our estimates suggest a larger inflation inertia than existing estimates, better capturing inflation persistence or adaptive inflation expectations, with the latter usually reflected in households survey indicators on inflation expectations. The overall reduced sampling uncertainty of the GBSE makes the policy implications derived from it more robust than their GMM counterparts.

The layout of the article is as follows. Section 2 proposes a new identification strategy for the NKPC. Section 3 introduces the generalized spectrum based estimation method. Section 4 reports the empirical results for the NKPC, and Section 5 concludes. Mathematical proofs are gathered in an Appendix at the end of the article. To save notation, throughout the article we drop almost surely from equalities involving conditional means.

\section{Identification strategy}

This section proposes a new identification strategy for the NKPC, which exploits nonlinear variation in inflation dynamics. We characterize nonparametric identification in the NKPC based on lagged instruments and conditional moments, rather than unconditional ones, and propose new tests for identification of the hybrid NKPC based on our characterization.

\subsection{Characterization of the NKPC identification}

For the study of the short-run inflation dynamics, researchers have actively sought to empirically identify the hybrid version of the NKPC, specifying the inflation rate at the current period as a linear function of the lagged value of inflation, the price-setters' rational forecast of the future inflation at the next period, and the current state of real economic activities:

$$
\pi_{t}=\gamma_{0}+\gamma_{b} \pi_{t-1}+\gamma_{f} E\left[\pi_{t+1} \mid I_{t}\right]+\lambda x_{t}+u_{t}
$$

where $\pi_{t}$ is the inflation rate at period $t, E\left[\pi_{t+1} \mid I_{t}\right]$ is the expected inflation rate at period $t+1$ conditioning on the information set $I_{t}$ available to the price-setters up to time $t, x_{t}$ is a measure of real marginal costs, and $u_{t}$ is an unobserved cost-push shock, which is not predictable at period $t-1$, i.e. $E\left[u_{t} \mid \mathcal{F}_{t-1}\right]=0$, with $\mathcal{F}_{t-1}$ the $\sigma$-field generated by $I_{t-1}$. Notice that model (4) is usually referred to as the "semi-structural" specification in the sense that the parameters $\gamma_{b}, \gamma_{f}$, and $\lambda$ are functions of the so-called "deep" parameters characterizing economic agents' behavior, see Galí and Gertler (1999) and references therein. 
An immediate obstacle to estimate the New Keynesian price equation (4) comes from the fact that the price-setters' inflation forecast $E\left[\pi_{t+1} \mid I_{t}\right]$ is not observed by the econometrician. To deal with this issue, previous studies substitute $E\left[\pi_{t+1} \mid I_{t}\right]$ in (4) with $\pi_{t+1}$ to obtain the equation

$$
\pi_{t}=\gamma_{0}+\gamma_{b} \pi_{t-1}+\gamma_{f} \pi_{t+1}+\lambda x_{t}+e_{t},
$$

where $e_{t}:=u_{t}-\gamma_{f}\left(\pi_{t+1}-E\left[\pi_{t+1} \mid I_{t}\right]\right)$. In the equation (5), $\pi_{t+1}$ is endogenous, and one can estimate the parameters by exploiting the moment conditions

$$
E\left[\left(\pi_{t}-\gamma_{0}-\gamma_{b} \pi_{t-1}-\gamma_{f} \pi_{t+1}-\lambda x_{t}\right) \mathbf{Z}_{t-1}\right]=0,
$$

provided a vector of valid instruments $\mathbf{Z}_{t-1}$ is available.

Galí and Gertler (1999) and subsequent researchers consider as instruments lagged variables dated $t-1$ or earlier, and treat $x_{t}$ as endogenous. ${ }^{2}$ Other studies in the literature, however, have assumed that $x_{t}$ is exogeneous, so it can used as a valid instrument, see, e.g., Nason and Smith (2008a). In this article, we consider the more difficult case where $x_{t}$ is assumed to be endogenous, although unreported empirical results suggested that the level of endogeneity in $x_{t}$ is not significant to affect our main results.

Another difficulty in estimating the NKPC is to procure an appropriate proxy for marginal cost $x_{t}$. Instead of using the output gap, which leads to negative estimated coefficients of $\lambda$, Galí and Gertler (1999) and Gali et al. (2001) suggest exploiting labor income share as a proxy for real marginal costs $x_{t}$. We follow this suggestion in our application below.

Despite the success of the hybrid NKPC of Galí and Gertler (1999), criticisms from econometric aspects have been raised with respect to the robustness of the GMM estimation results. It is by now well established that the presence of weak instruments renders GMMbased inferences on NKPC highly unreliable, as investigated in Staiger and Stock (1997), Wang and Zivot (1998), Stock et al. (2002), and Kleibergen (2002), among others. Stimulated by the weak instrument literature, a line of recent studies including Ma (2002), Mavroeidis (2005), Dufour et al. (2006), Nason and Smith (2008a) and Kleibergen and Mavroeidis (2009) employ identification-robust methods to find that the NKPC is weakly identified, suggesting that the potential pitfalls of weak identification in GMM estimation of the hybrid NKPC should be taken into consideration. The conclusion from this literature is that there is a substantial amount of sampling uncertainty in GMM estimates, and that new identification approaches are needed to reach an empirical consensus (cf. Mavroeidis, Plagborg-Møller, and Stock (2014)).

\footnotetext{
${ }^{2}$ Gali et al. (2001) justify the use of lagged value of $x_{t}$ as follows: First, considering the potentially large measurement error for marginal costs, it is reasonable to assume that the error is uncorrelated with past information. Second, not all current information may be available to the price-setters.
} 
This article proposes an alternative identification strategy of the NKPC, which is different from the standard linear GMM approach. Formally, we consider the conditional moment restriction obtained from (4) with $E\left[u_{t} \mid I_{t-1}\right]=0$ :

$$
E\left[\pi_{t}-\gamma_{0}-\gamma_{b} \pi_{t-1}-\gamma_{f} \pi_{t+1}-\lambda x_{t} \mid I_{t-1}\right]=0
$$

We write this equation as a regression model with infeasible exogenous regressors

$$
\pi_{t}=\boldsymbol{\theta}_{0}^{\prime} \mathbf{X}_{t}^{e}+\varepsilon_{t}
$$

where $\boldsymbol{\theta}_{0}:=\left(\gamma_{0}, \gamma_{b}, \gamma_{f}, \lambda\right)^{\prime}, \mathbf{X}_{t}^{e}:=\left(1, \pi_{t-1}, E\left[\pi_{t+1} \mid I_{t-1}\right], E\left[x_{t} \mid I_{t-1}\right]\right)^{\prime}$ and $\varepsilon_{t}:=\pi_{t}-E\left[\pi_{t} \mid I_{t-1}\right]$. Notice that $\left\{\varepsilon_{t}\right\}$ is a martingale difference sequence by construction. ${ }^{3}$ By standard least squares theory, $\boldsymbol{\theta}_{0}$ is identified in (8) if and only if $\mathbf{X}_{t}^{e}$ satisfies that $E\left[\mathbf{X}_{t}^{e} \mathbf{X}_{t}^{e \prime}\right]$ is full rank. Unfortunately, $\mathbf{X}_{t}^{e}$ is not observable, which makes the rank identification condition hard to check in practice, and the main contribution of this section is to characterize this rank identification condition in terms of conditional moment restrictions involving observable variables. We assume throughout that $\operatorname{Var}\left(\pi_{t-1}\right)>0$.

Our first result characterizes the singularity of $E\left[\mathbf{X}_{t}^{e} \mathbf{X}_{t}^{e \prime}\right]$ (i.e. lack of identification) in terms of the two conditional moments

$$
E\left[x_{t}+\beta_{0}+\beta_{1}\left(\pi_{t+1}-x_{t}\right)-\beta_{2} \pi_{t-1} \mid I_{t-1}\right]=0 \text { for some } \beta_{0}, \beta_{1}, \beta_{2} \in \mathbb{R}
$$

and

$$
E\left[\left(\pi_{t+1}-x_{t}\right)-\beta_{3}-\beta_{4} \pi_{t-1} \mid I_{t-1}\right]=0 \text { for some } \beta_{3}, \beta_{4} \in \mathbb{R} .
$$

Conditions (9) or (10) imply lack of identification since they entail perfect (conditional) multicollinearity in the reduced-form regression (8). Surprisingly, the reciprocal is also true, as shown in the following proposition, the proof of which is provided in the Appendix.

Proposition 1. $\boldsymbol{\theta}_{0}$ is not identified from (7) if and only if (9) or (10) holds.

Proposition 1 provides us with a formal test for identification of the NKPC that can be used to empirically assess identification with observed data. That is, suppose that we aim to test the null hypothesis of lack of identification of the NKPC,

$$
H_{0}: \boldsymbol{\theta}_{0} \text { is not identified from }(7)
$$

against the alternative, say $H_{1}$, of identification. By Proposition 1, this can be equivalently

\footnotetext{
${ }^{3}$ In contrast, the GMM error $e_{t}$ in $(5)$ is not adapted to the information at period $t$ due to the inclusion of the next period inflation $\pi_{t+1}$, which may cause first-order autocorrelation in the error $\left\{e_{t}\right\}$.
} 
written as

$$
H_{0}:(9) \text { or }(10) \text { holds, }
$$

which is tested against its negation.

Before constructing a test statistic, we first need to check if the $\beta$ 's are identified in (9) or (10), as their identification is often a necessary regularity condition for existing tests. It is apparent that the parameter $\beta_{3}$ and $\beta_{4}$ are identified from (10), since $\operatorname{Var}\left(\pi_{t-1}\right)>0$. However, in assessing the identifiability of $\left(\beta_{0}, \beta_{1}, \beta_{2}\right)$ in $(9)$, it is useful to investigate the link between (9) and (10), which is done in the next result.

Proposition 2. $\left(\beta_{0}, \beta_{1}, \beta_{2}\right)$ is not identified from (9) if and only if (10) holds.

Proposition 1 and Proposition 2 suggest an empirical strategy to test for identification in the NKPC. First, we use existing parametric or nonparametric testing methods to check if (10) holds. If the tests fail to reject (10), then we conclude that $\boldsymbol{\theta}_{0}$ is not identified. If (10) does not hold, then we turn to check the validity of (9). In this case, Proposition 2 implies that $\left(\beta_{0}, \beta_{1}, \beta_{2}\right)$ is identified in (9), thus allowing us to apply standard methods to test (9). If it turns out that (9) holds, then we conclude $\boldsymbol{\theta}_{0}$ is not identified. Hence, we can utilize conventional testing approaches, coupled with simple methods to control size distortions from sequential testing, such as Bonferroni corrections, to check for identification of the NKPC.

We can interpret equation (10) as a linearity restriction as follows. Define the dependent variable $Y_{t+1}=\pi_{t+1}-x_{t}$. Then, an implication of (10) is

$$
E\left[Y_{t+1} \mid \pi_{t-1}\right]=\beta_{3}+\beta_{4} \pi_{t-1} \text { for some } \beta_{3}, \beta_{4} \in \mathbb{R}
$$

This linearity restriction can be empirically tested. Indeed, our tests below reject (12), hence (10), with U.S data. Nonlinearity in inflation dynamics and related variables is consistent with existing economic theories such as the opportunistic disinflation idea of Orphanides and Wilcox (2002), models of monetary policies with regime switching in Davig and Leeper (2007, 2008), or nonlinear monetary policy rules in Dolado et al. (2004), among others. To illustrate this point, we consider an example adapted from Davig and Leeper (2008).

Example. Consider the New Keynesian Model with Endogenous Regime Switching Monetary Policy:

$$
\begin{aligned}
\pi_{t} & =\gamma_{b} \pi_{t-1}+\gamma_{f} E\left[\pi_{t+1} \mid I_{t}\right]+\lambda x_{t} \\
x_{t} & =E\left[x_{t+1} \mid I_{t}\right]-\sigma^{-1}\left(i_{t}-E\left[\pi_{t+1} \mid I_{t}\right]\right) \\
i_{t} & =\alpha\left(\pi_{t-1}\right) \pi_{t}
\end{aligned}
$$


where $i_{t}$ is the short-term nominal interest rate set by the central bank, and the statedependent coefficient is $\alpha\left(\pi_{t-1}\right)=1\left(\pi_{t-1}<\pi^{*}\right) \alpha_{0}+1\left(\pi_{t-1} \geq \pi^{*}\right) \alpha_{1}$, where $1(\cdot)$ is the indicator function, $\pi^{*}$ is a threshold point, which defines the regime, and $1<\alpha_{0}<\alpha_{1}$. Assuming $x_{t}$ follows an exogenous first-order autoregression $x_{t}=\rho x_{t-1}+v_{t}, v_{t} \sim i . i . d$., which seems to be consistent with dynamics of output gap, one can easily show that the conditional expectation for the future inflation is a nonlinear function of conditioning variables $\left(\pi_{t-1}, \pi_{t}, x_{t}\right)$ given by

$$
E\left[\pi_{t+1} \mid \pi_{t-1}, \pi_{t}, x_{t}\right]=\sigma(1-\rho) x_{t}+\alpha\left(\pi_{t-1}\right) \pi_{t}
$$

This in turn implies the equation

$$
E\left[Y_{t+1} \mid \pi_{t-1}\right]=b E\left[x_{t} \mid \pi_{t-1}\right]+\alpha\left(\pi_{t-1}\right) E\left[\pi_{t} \mid \pi_{t-1}\right]
$$

where, recall $Y_{t+1}=\pi_{t+1}-x_{t}$, and $b=\sigma(1-\rho)-1$. Even in the unlikely event that both $E\left[x_{t} \mid \pi_{t-1}\right]$ and $E\left[\pi_{t} \mid \pi_{t-1}\right]$ are linear functions of $\pi_{t-1}$, the conditional mean $E\left[Y_{t+1} \mid \pi_{t-1}\right]$ will be a nonlinear function of $\pi_{t-1}$ if $\alpha_{0} \neq \alpha_{1}$.

Similarly, the equations above imply the conditional moment

$$
E\left[Y_{t+1}-c x_{t}-\gamma_{b} \alpha\left(\pi_{t-1}\right) \pi_{t-1}-\gamma_{f} \alpha\left(\pi_{t-1}\right) \pi_{t+1} \mid I_{t-1}\right]=0
$$

for $c=b+\lambda$. Again, this implication of the regime switching model is in general incompatible with the conditional moment (9) when $\alpha_{0} \neq \alpha_{1}{ }^{4}$

Thus, endogenous regime switching is a potential source of nonlinearity in the reduced forms that, according to our results, may help in identifying the NKPC. We use these insights to develop simple parametric tests for testing (9) and (10) based on threshold models in our application. We also propose nonparametric tests that confirm the empirical results obtained from the parametric tests. Specific details on the nonparametric tests used to check (9) and (10) are provided in the Appendix.

\section{Generalized Band Spectrum Estimation}

Having studied identification of the NKPC, this section introduces a novel estimator that accounts for nonlinear variation in inflation dynamics, and which follows our identification strategy. The new estimator is called the Generalized Band Spectrum Estimator (GBSE). The estimator is simple to use because it has a closed-form solution, so no optimization

\footnotetext{
${ }^{4}$ When $\beta_{1}$ in (9) is not zero, (9) can be written as $E\left[Y_{t+1}-c_{0}-c_{1} x_{t}-c_{2} \pi_{t-1} \mid I_{t-1}\right]=0$, for some $c_{0}, c_{1}$ and $c_{2}$.
} 
is needed. Unlike standard GMM estimators, the GBSE uses all the information from the conditional moments, for all $j \geq 1$,

$$
E\left[\pi_{t}-\gamma_{0}-\gamma_{b} \pi_{t-1}-\gamma_{f} \pi_{t+1}-\lambda x_{t} \mid \mathbf{Z}_{t-j}\right]=0
$$

where $\mathbf{Z}_{t-j}$ are selected instruments in $I_{t-1}$. Accounting for all lags is important given the high persistence of inflation and related variables (Cogley and Sbordone (2008)). Additionally, the GBSE permits a focus on a particular set of frequencies, such as those measuring short-run dynamics, which is important in applications such as the NKPC. The GBSE is a single-equation estimator, and as such is robust to the misspecification of the first-stage or other structural equations in a full system. For example, a full information estimator of the endogenous regime switching model might lead to more efficient estimates of the NKPC parameters than the GBSE, but at the cost of being inconsistent if the dynamics of marginal cost and interest rates are misspecified. In contrast, the GBSE is robust to these misspecifications, as long as the identification condition above holds.

The GBSE for a specific frequency band $\left[a_{1}, a_{2}\right], a_{1}, a_{2} \in[0,1]$, and data $\left\{\pi_{t}, \pi_{t+1}, x_{t}, \mathbf{Z}_{t}\right\}_{t=0}^{n}$ is a weighted least squares estimator given by

$$
\widehat{\boldsymbol{\theta}}_{G B S E}=\left(\mathbf{X}^{\prime} \widetilde{\mathbf{W}} \mathbf{X}\right)^{-1}\left(\mathbf{X}^{\prime} \widetilde{\mathbf{W}} \boldsymbol{\pi}\right),
$$

where $\mathbf{X}$ is the classical design matrix with rows $\left(\mathbf{X}_{1}^{\prime}, \mathbf{X}_{2}^{\prime}, \ldots, \mathbf{X}_{n}^{\prime}\right)^{\prime}, \mathbf{X}_{t}:=\left(1, \pi_{t-1}, \pi_{t+1}, x_{t}\right)^{\prime}$, $\boldsymbol{\pi}:=\left(\pi_{1}, \pi_{2}, \ldots, \pi_{n}\right)^{\prime}$, and $\widetilde{\mathbf{W}}$ is a matrix with $(t, s)$ entry

$$
(\widetilde{\mathbf{W}})_{t, s}=\sum_{j=1}^{t} \sum_{k=1}^{s}\left(\frac{1}{n_{j}^{1 / 2}(j \pi)}\right)\left(\frac{1}{n_{k}^{1 / 2}(k \pi)}\right) \exp \left\{-\frac{1}{2}\left|\mathbf{Z}_{t-j}-\mathbf{Z}_{s-k}\right|^{2}\right\} \Upsilon_{j k}\left(a_{1}, a_{2}\right)
$$

where $\Upsilon_{j k}\left(a_{1}, a_{2}\right):=\int_{\left[a_{1}, a_{2}\right]}\{2 \sin (j \pi \omega) \sin (k \pi \omega)\} d \omega$ and $n_{j}=n-j+1$.

For a formal derivation of this estimator using Hilbert space arguments in the frequency domain see the mathematical Appendix. Unlike GMM and related linear methods, the GBSE accounts for nonlinear dependence. Specifically, linear methods, such as those developed by Hannan (1965), Hannan (1967), Engle (1974) and Berkowitz (2001) exploit linear correlations, as measured by the moments

$$
E\left[\left(\pi_{t}-\gamma_{b} \pi_{t-1}-\gamma_{f} \pi_{t+1}-\lambda x_{t}\right) \mathbf{Z}_{t-j}\right]=0
$$

The GBSE effectively exploits all the information from the conditional moments (13) by 
using the continuum of moments

$$
\gamma_{j}\left(\boldsymbol{\theta}_{0}, \mathbf{z}\right):=E\left[\left(\pi_{t}-\gamma_{b} \pi_{t-1}-\gamma_{f} \pi_{t+1}-\lambda x_{t}\right) \exp \left(i \mathbf{z}^{\prime} \mathbf{Z}_{t-j}\right)\right]=0
$$

for all $\mathbf{z}$, where $\mathbf{z}$ is a vector of the same dimension as $\mathbf{Z}_{t}$, and $i=\sqrt{-1}$. The classical (cross)spectrum corresponding to (15) is zero at the true parameter value $\boldsymbol{\theta}_{0}=\left(\gamma_{b}, \gamma_{f}, \lambda\right)^{\prime}$. This is the basis for classical band spectrum estimators. Similarly, the generalized spectrum introduced by Hong (1999) and based on the generalized covariances $\left\{\gamma_{j}\left(\boldsymbol{\theta}_{0}, \mathbf{z}\right)\right\}_{j=1}^{\infty}$ is flat at the true value, which is the basis for the GBSE. This estimator extends the estimator of Dominguez and Lobato (2004) and Escanciano (2017) to a spectral setting with an infinite number of lags, and is a nonlinear and nonparametric version of the well-known band spectrum estimator of Hannan (1967), Engle (1974) and Berkowitz (2001). Notice that by differentiating $\gamma_{j}\left(\boldsymbol{\theta}_{0}, \mathbf{z}\right)$ at $\mathbf{z}=\mathbf{0}$, we recover the moment (15), so $\gamma_{j}\left(\boldsymbol{\theta}_{0}, \mathbf{z}\right)$ contains information from first and higher order derivatives (i.e. nonlinearities as well). The GBES integrates all $\mathbf{z}$ in $\gamma_{j}\left(\boldsymbol{\theta}_{0}, \mathbf{z}\right)$, and all frequencies in $\left[a_{1}, a_{2}\right]$, and as such, it contains information from nonlinear moments, e.g. $E\left[\left(\pi_{t}-\gamma_{b} \pi_{t-1}-\gamma_{f} \pi_{t+1}-\lambda x_{t}\right) \pi_{t-1}^{2}\right]=0$, and from all lags. A formal derivation of the GBES is given in the Appendix.

The following theorem states the consistency and asymptotic normality of the GBSE. First, set $\Omega:=\operatorname{diag}\left(\varepsilon_{1}^{2}, \varepsilon_{2}^{2}, \ldots, \varepsilon_{n}^{2}\right)$ and define the probabilistic limits $(p \lim )$

$$
\begin{aligned}
& \boldsymbol{\Sigma}:=p \lim _{n \rightarrow \infty} \mathbf{X}^{\prime} \mathbf{W X} / n^{2} \\
& \boldsymbol{\Lambda}:=p \lim _{n \rightarrow \infty} \mathbf{X}^{\prime} \mathbf{W} \boldsymbol{\Omega} \mathbf{W X} / n^{3},
\end{aligned}
$$

which are well-defined under mild moment conditions.

Theorem 1. Under Assumption 1 in the Appendix, $\widehat{\boldsymbol{\theta}}_{G B S E}$ is consistent and asymptotically normal, i.e.

$$
\sqrt{n}\left(\widehat{\boldsymbol{\theta}}_{G B S E}-\boldsymbol{\theta}_{0}\right) \rightarrow_{d} N\left(0, \boldsymbol{\Sigma}^{-1} \boldsymbol{\Lambda} \boldsymbol{\Sigma}^{-1}\right) .
$$

The proof of Theorem 1 is gathered in the Appendix. A natural estimator for the asymptotic variance of the GBSE is given by $\widehat{\mathbf{V}}:=n^{-1} \widehat{\boldsymbol{\Sigma}}^{-1} \widehat{\Lambda} \widehat{\boldsymbol{\Sigma}}^{-1}$, where $\widehat{\boldsymbol{\Sigma}}=\left(\mathbf{X}^{\prime} \mathbf{W X}\right) / n^{2}$ and $\widehat{\Lambda}=$ $\left(\mathbf{X}^{\prime} \mathbf{W} \widehat{\boldsymbol{\Omega}} \mathbf{W X}\right) / n^{3}$, with $\widehat{\boldsymbol{\Omega}}=\operatorname{diag}\left(\widehat{\varepsilon}_{1}^{2}, \widehat{\varepsilon}_{2}^{2}, \ldots, \widehat{\varepsilon}_{n}^{2}\right)$ where $\widehat{\varepsilon}_{t}:=\pi_{t}-\widehat{\boldsymbol{\theta}}_{G B S E}^{\prime} \mathbf{X}_{t}$. The consistency of $\widehat{\mathbf{V}}$ follows from the law of large numbers using the fact that $\varepsilon_{t}$ in (8) is a martingale difference sequence with respect to $\mathcal{F}_{t-1}$. 


\section{Application to hybrid NKPC}

This section applies our proposed procedures to identifying and estimating the hybrid NKPC with U.S. quarterly data. We investigate if exploiting nonlinear variation would be conducive to identifying the NKPC, as theoretically justified by Proposition 1 above.

\subsection{Data}

For our empirical analysis, all the U.S. quarterly data we use are obtained from the 1998 and 2012 vintage datasets provided by Mavroeidis, Plagborg-Møller, and Stock (2014). ${ }^{5}$ For identification tests, we use 2012 vintage data from 1970Q1 to 2011Q4. Then, to fairly compare our GBSE estimation with GMM estimation in Mavroeidis, Plagborg-Møller, and Stock (2014), we truncate the sample and use the data from 1970Q1 to 1998Q1. Inflation $\left(\pi_{t}\right)$ is defined as the log difference in the gross domestic product (GDP) deflator, while marginal costs $\left(x_{t}\right)$ are measured by the non-farm business labor share, as suggested by Galí and Gertler (1999). The details about instruments variables will be discussed in the following sections.

\subsection{Identification}

In this section we provide empirical evidence that supports point-identification of the NKPC with our identification strategy. First, we consider parametric tests for (9) and (10) motivated from the endogenous regime switching model example. Second, we implement nonparametric bootstrap tests that confirm the results of the parametric tests.

\subsubsection{Parametric Identification Tests}

Our identification analysis suggests first testing for the conditional moment (10). Any nonlinearity test would be valid for this, but a simple test that is well motivated from the endogenous regime switching example is to run the following simple regression model

$$
Y_{t+1}=\delta_{0}+\delta_{1} \pi_{t-1}+\delta_{2} \cdot 1\left(\pi_{t-1} \geq \pi^{*}\right)+\delta_{3} \cdot 1\left(\pi_{t-1} \geq \pi^{*}\right) \cdot \pi_{t-1}+\varepsilon_{1 t},
$$

where $\pi^{*}$ is set to 1.75 (other values between 1.5 and 2 led to the same conclusions). We calibrate $\pi^{*}$ rather than estimate it because this parameter is not identified under one of our null hypothesis, $H_{0}: \delta_{2}=\delta_{3}=0$, which leads to non-standard inference. We develop below a nonparametric test that overcomes the complications associated to the lack of identification

\footnotetext{
${ }^{5}$ The dataset used in Mavroeidis, Plagborg-Møller, and Stock (2014) can be downloaded from: https://www.aeaweb.org/articles?id=10.1257/jel.52.1.124.
} 
and non-standard inferences. Model (16) is a nonlinear (in variables) regression model when $\delta_{2}$ or $\delta_{3}$ are non-zero. Parametric tests for significance of $\delta_{2}$ or $\delta_{3}$ can be used to testing (10). Table 1 reports OLS estimates for the $\delta^{\prime} s$ and their corresponding t-test statistics. We reject the hypotheses that $H_{0}: \delta_{2}=0$ and $H_{0}: \delta_{3}=0$ at $5 \%$ nominal level. Then, we use F-test for the joint hypothesis $H_{0}: \delta_{2}=\delta_{3}=0$. Table 1 also shows that this hypothesis is strongly rejected at all commonly used nominal levels. Hence, both test statistics imply that (10) is rejected.

\section{TABLE 1 ABOUT HERE}

To test for $(9)$, we assume that $\beta_{1} \neq 0$ and consider the regression model

$$
Y_{t+1}=\alpha_{0}+\alpha_{1} x_{t}+\alpha_{2} \pi_{t-1}+\alpha_{3} \cdot 1\left(\pi_{t-1} \geq \pi^{*}\right)+\alpha_{4} \cdot 1\left(\pi_{t-1} \geq \pi^{*}\right) \cdot \pi_{t-1}+\varepsilon_{2 t},
$$

where again we set $\pi^{*}=1.75$. Now the hypotheses of interest are $H_{0}: \alpha_{3}=0, H_{0}: \alpha_{4}=0$ or $H_{0}: \alpha_{3}=\alpha_{4}=0$. Table 2 reports OLS estimates for the $\alpha^{\prime} s$ and their corresponding t-test and F-test statistics. We strongly reject the linearity hypotheses, and hence (9).

\section{TABLE 2 ABOUT HERE}

In summary, the parametric tests of this section, in combination with our theoretical results in Proposition 1 and Proposition 2, provide empirical support for the point identification of the NKPC. The parametric tests above are simple to interpret, but they are not consistent against all deviations from linearity and depend on the assumption that $\beta_{1} \neq 0$ in the case of (9). To check the robustness of the implications based on parametric tests we consider consistent nonparametric tests in the next subsection.

\subsubsection{Nonparametric Identification Tests}

Evidence of nonlinearity of $E\left[Y_{t+1} \mid \pi_{t-1}\right]$ is evidence in favor of identification (Proposition $1)$. Figure 1 plots a nonparametric Gaussian kernel estimator of $E\left[Y_{t+1} \mid \pi_{t-1}\right]$ with a crossvalidated bandwidth $h=0.72$, in comparison with a linear fit. This plot suggests that a nonlinear fit, perhaps modelled as a threshold regime switching model, is more appropriate than a linear fit. Large values of lagged inflation tend to be associated with smaller values of future inflation than the linear model suggests. The linear model provides a good fit until an approximate turning point of $\pi^{*}=1.75$, after which the slope of the nonlinear fit becomes smaller than that suggested by the linear model. This is consistent with the positive and significant estimate of $\delta_{3}$ in Table 1 .

A nonparametric test of linearity based on the kernel estimator would very much depend 
on the value of the bandwidth considered. To address this issue, we modify the nonparametric bootstrap tests proposed in Escanciano (2006), which avoids bandwidth choices. These nonparametric bootstrap tests, which are developed in the Appendix, can be interpreted as nonlinear and nonparametric analogues of the first stage $F$-test that are commonly used in the literature of linear models, see Stock and Yogo (2005). We implement the bootstrap tests with 1000 bootstrap simulations. The obtained bootstrap $p$-values are zero for both hypotheses, (9) and (10), which suggests that at all conventional levels both (9) and (10) are rejected, supporting the conclusions from parametric tests and thus, the point-identification of the hybrid NKPC.

\section{FIGURE 1 ABOUT HERE}

\subsection{Baseline model estimation}

This section turns into estimation and applies the GBSE to the NKPC. Some empirical studies suggest that the relation between inflation and output seems more stable at highfrequencies. For instance, using U.S. quarterly data from 1950s to 2002, Zhu (2005) finds that the Phillips curve relationship is stable at high frequencies, but very unstable in the business cycle frequency and the low frequency range. Also, Assenmacher-Wesche and Gerlach (2008a,b) suggest that the high frequency component of inflation dynamics in the Euro area and Switzerland are mainly explained by output gap. Given this empirical evidence, we attempt to investigate the short-run inflation dynamics using our GBSE which exploits information in the high frequency band. ${ }^{6}$

Then, we compare the estimates obtained from our GBSE with those in Mavroeidis, Plagborg-Møller, and Stock (2014). These authors estimate the hybrid NKPC of Galí and Gertler (1999) using the continuously updated GMM approach of Hansen et al. (1996). They conclude that GMM estimation is subject to weak identification. To enhance the comparability between our GBSE estimator and the GMM estimator in Mavroeidis, Plagborg-Møller, and Stock (2014), we use the same model specification and vintage datasets and the same sample period (from 1970Q1 to 1998Q2) as those used in Mavroeidis, Plagborg-Møller, and Stock (2014). They use the 1998 and 2012 vintage datasets, respectively, and show that the revision of the data matter for the results.

With regard to the choice of instruments, we also follow Mavroeidis, Plagborg-Møller, and Stock (2014) and use four lags of inflation and two lags of the labor share, wage inflation, and quadratically-detrended output as instruments for our baseline model. In later subsections,

\footnotetext{
${ }^{6}$ Following Baxter and King (1999), we define these frequency bands as: the long-term cycle (more than 8 years), the medium-term cycle $(1.5 \sim 8$ years), and the short-term cycle band (less than 1.5 years).
} 
we will exploit instrumental variables that are also commonly used the literature, see, e.g., Galí and Gertler (1999), Dufour et al. (2006), Nason and Smith (2008a,b), including lagged values of the current inflation rate, real unit labor costs or labor share as proxy for marginal costs, and four key macroeconomic variables apparently associated with the overall inflation rates. These four macro variables consist of quadratically de-trended output gap $(y)$, the long-short interest rate differential $(t s)$, wage inflation $(w i)$ and commodity price inflation $(c p)$. Notice that, in line with the majority of the literature, we treat the forcing variable $x_{t}$ as endogenous, which implies that only the lags of $x_{t}$ are included as instruments.

The regression is an non-demeaned hybrid NKPC model as follows:

$$
\pi_{t}=\gamma_{0}+\gamma_{b} \pi_{t-1}+\gamma_{f} E\left[\pi_{t+1} \mid I_{t}\right]+\lambda x_{t}+u_{t}
$$

Table 3 presents the GBSE estimates for the high frequency band $\left[a_{1}, a_{2}\right]=\left[\frac{1}{3}, 1\right]$ and with the same instruments as those considered for the continuously updated GMM of Mavroeidis, Plagborg-Møller, and Stock (2014). The GBSE significantly differs from the GMM estimator in several dimensions. First, the GBSE of $\lambda$ is statistically significant even when we use revised data released in 2012, while its counterpart obtained from GMM estimation becomes statistically insignificant (and smaller) if we use vintage data released in 2012 for estimation (Rudd and Whelan (2007)). Moreover, our GBSE estimation shows that both forwardlooking and backward-looking terms are equally important, while the GMM estimation shows that the forward-looking term dominates. Lastly, the standard errors for $\hat{\gamma}_{f}$ and $\hat{\gamma}_{b}$ obtained from our GBSE are much smaller than their counterparts in GMM estimation. This gain in precision may be explained by the more efficient use of information from the conditional moments by the GBSE, relative to the standard GMM estimator which is based on a finite set of unconditional moments that are subject to weak identification.

\section{TABLE 3 ABOUT HERE}

Our estimates have important implications for policy. On one hand, the larger values obtained for the coefficient of marginal cost with the GBSE suggest a more important role for monetary policy than their GMM counterparts. On the other hand, our estimate for the coefficient of expected future inflation, although quantitatively important and hence suggestive of a significant role for expectations management and communications as tools of monetary policy, is relatively smaller than previous GMM estimates. The larger values obtained for inflation inertia have important implications for design of optimal monetary policy, as shown in Schmitt-Grohé and Uribe (2008). 


\subsection{Robustness checks}

This subsection conducts robustness checks to validate our empirical findings from the GBSE by changing the instruments, specification of the forcing variable and specification of the dependent variable, respectively.

Table 4 presents robustness checks by changing the specification of the instrument variables. The instrument Z1 consists of 4 lags of inflation, labor share, output gap, yield spread, wage inflation and commodity inflation. The instrument Z2 consists of 4 lags of inflation and 3 lags of labor share. Both instruments Z1 and Z2 are based on Mavroeidis, Plagborg-Møller, and Stock (2014). Table 4 shows that the estimates of $\lambda$ are still significant at $5 \%$ nominal level. Moreover, the magnitude of these estimates are close to the those obtained from the benchmark model.

Table 5 presents robustness checks by changing the specification of the forcing variable. The alternative specification of the forcing variable, X1, is based on Nason and Smith $(2008 \mathrm{a})^{7}$ while X2 is based on Sbordone $(2005)^{8}$. Table 5 shows that although the magnitude of $\hat{\lambda}$ seems to be smaller that in the benchmark model, it is still statistically significant at $5 \%$ nominal level.

Table 6 presents robustness checks by changing the specification of the dependent variable, $\pi_{t}$. In the benchmark model $\pi_{t}$ is the implied GDP deflator. Here, we replace implied GDP deflator with consumer price index (CPI) and price index of personal consumption expenditure (PCE), respectively. While the implied GDP deflator measures the price level of domestic goods, CPI and PCE includes both domestic and foreign goods bought by consumers. Moreover, there is also difference between CPI and PCE since each measure assigns different weights to different kinds of goods. However, the estimated coefficient for the output gap, $\hat{\lambda}$, still remains statistically significant across different specification despite the difference among these price indices.

Overall, Tables 4, 5 and 6 show that our GBSE remains stable across different model specifications in both versions of vintage data. The stability of the GBSE contrasts with the well-documented instability of GMM and related linear estimators.

\section{TABLES 4, 5 and 6 ABOUT HERE}

\footnotetext{
${ }^{7}$ In Nason and Smith (2008a), the approximate of real marginal cost, X1, is defined as $X 1=100(1+$ q) $\ln \left(C O M P N F B_{t} / O P H N F B_{t}\right)-100 P_{t}$, where $q$ is a function of the steady-state markup and labor share parameter in the firms production function, COMPNFB is an index of the nominal wage and OPHNFB is an index of the average product of labor.

${ }^{8}$ In Sbordone (2005), the approximate of real marginal cost, X2, is defined as $X 2=s_{t}+\delta_{0}\left(\mathrm{~d} h_{t}-\right.$ $\left.\delta_{1} E_{t} \mathrm{~d} h_{t+1}\right)$, where $s_{t}$ is the (log of) average real marginal cost in the economy, the term in brackets represent the expected deviation of future hours growth from current growth, and the coefficient $\delta_{0}$ measures the curvature of the adjustment cost function.
} 


\section{Concluding remarks}

This article proposes a new framework for identifying and estimating the hybrid New Keynesian Phillips curve of Galí and Gertler (1999), exploiting nonlinear variation of unknown functional form in inflation dynamics. An appealing property of our identification strategy is that the strength of the identification can be quantitatively assessed by suitable tests of conditional moment restrictions. For the NKPC estimation, we propose a generalized band-spectrum estimator that is able to capture nonlinear variation as well as to incorporate information on the serial dependence from all lags in the sample, while permitting a focus on short run dynamics, where the relationship between inflation and marginal cost is stronger.

Empirical findings from this study are summarized as follows. First, our methods for assessing identification provide empirical evidence of point-identification in U.S. postwar data, thereby suggesting that the weak identification of linear methods are resolved by introducing additional layers of information from nonlinear dynamics. We find that the forward-looking component and the inflation inertia are both quantitatively important and statistically significant in explaining U.S. inflation dynamics, with smaller standard errors than those reported in studies based on GMM. Our coefficient of marginal cost is significantly larger than those obtained by GMM.

We envision other applications of the methodological contributions of this article. For instance, one may employ the proposed approach in other important macroeconomic applications involving rational expectations where the weak identification problem arises, see, e.g., the Euler equation for output or the monetary policy reaction function (Mavroeidis (2010)). It is also possible to extend the proposed identification and estimation strategies to multiple RE models.

Our identification analysis has also implications for inflation forecasting. Although the GMM estimation literature of the NKPC has shown little linear predictability of future inflation once we control for lagged inflation, our identification results suggest potential nonlinear predictability of future inflation. It should be of interest to extract the implicit nonlinear predictors from our nonparametric tests following similar ideas to those exposed in Escanciano and Mayoral (2010). We shall investigate these extensions of our methods and other applications in the future. 


\section{Appendix}

\subsection{Proofs of identification results}

Proof of Proposition 1: If (9) or (10) holds, then $\boldsymbol{\theta}_{0}$ is not identified, since $\boldsymbol{\theta}_{1}:=\boldsymbol{\theta}_{0}+$ $\left(\beta_{0},-\beta_{2}, \beta_{1}, 1-\beta_{1}\right)^{\prime}$ or $\boldsymbol{\theta}_{2}:=\boldsymbol{\theta}_{0}+\left(-\beta_{3},-\beta_{4},+1,-1\right)^{\prime}$ also satisfies (7). We now prove that if $\boldsymbol{\theta}_{0}$ is not identified, then (9) or (10) holds. That is, suppose that $\boldsymbol{\theta}_{1}:=\left(\gamma_{1}, \gamma_{b 1}, \gamma_{f 1}, \lambda_{1}\right)^{\prime}$, which is different from $\boldsymbol{\theta}_{0}$, also satisfies (7). Then,

$$
E\left[\left(\gamma_{0}-\gamma_{1}\right)-\left(\gamma_{b}-\gamma_{b 1}\right) \pi_{t-1}+\left(\gamma_{f}-\gamma_{f 1}\right) \pi_{t+1}+\left(\lambda-\lambda_{1}\right) x_{t} \mid I_{t-1}\right]=0 \text { a.s. }
$$

We now consider an exhaustive list of cases regarding the values that the coefficients $c_{1}:=$ $\left(\gamma_{f}-\gamma_{f 1}\right)$ and $c_{2}:=\left(\lambda-\lambda_{1}\right)$ can take: (i) $c_{1}=c_{2}=0$; (ii) $c_{1} \neq 0$ but $c_{2}=0$; (iii) $c_{1}=0$ but $c_{2} \neq 0$; (iv) $c_{1} \neq 0, c_{2} \neq 0$, and $c_{1}+c_{2} \neq 0$; and (v) $c_{1} \neq 0, c_{2} \neq 0$, and $c_{1}+c_{2}=0$. Case (i) leads to a contradiction, because $\pi_{t-1} \in I_{t-1}, \operatorname{Var}\left[\pi_{t-1}^{2}\right]>0$, and then $\gamma_{0}=\gamma_{1}$ and $\gamma_{b}=\gamma_{b 1}$ must hold. In both, (ii) and (iii), we can divide by the non-zero coefficient and transform (19) into (9) with $\beta_{1}=1$ and $\beta_{1}=0$, respectively. In case (iv) we can divide (19) by $c_{1}+c_{2}$ to obtain (9) with $\beta_{1}=c_{1} /\left(c_{1}+c_{2}\right)$. Finally, in (v) we can divide (19) by $c_{1}$ to obtain (10).

Proof of Proposition 2: If (10) holds then there is perfect multicollinearity in (9) and identification fails. Suppose that identification of (9) fails, and $\left(b_{0}, b_{1}, b_{2}\right)$ also satisfies this moment condition, and it is different from $\left(\beta_{0}, \beta_{1}, \beta_{2}\right)$. Then, there exists some $\left(c_{0}, c_{1}, c_{2}\right) \neq$ $(0,0,0)$ such that $E\left[c_{0}+c_{1}\left(x_{t}-\pi_{t+1}\right)+c_{2} \pi_{t-1} \mid I_{t-1}\right]=0$. Note that $c_{1}$ cannot be zero, since $\operatorname{Var}\left(\pi_{t-1}\right)>0$. Dividing by $c_{1}$, we obtain the result.

\subsection{Asymptotic distribution theory for the GBSE}

This section formally introduces the GBSE. For simplicity of exposition, we consider the full spectrum case $\left[a_{1}, a_{2}\right]=[0,1]$. Let us define $\boldsymbol{\eta}:=\left(\omega, \mathbf{z}^{\prime}\right)^{\prime} \in[0,1] \times \mathbb{R}^{d}$ and $\Psi_{j}(\omega):=$ $\sqrt{2} \sin (j \pi \omega) / j \pi$. Under $E\left[\left|\pi_{t}\right|^{2}\right]<\infty$, it can be shown that the following integrated moment is well-defined (as an element in a suitable Hilbert space)

$$
h_{\pi}(\boldsymbol{\eta}):=E\left[\pi_{t} q_{t-1}(\boldsymbol{\eta})\right]
$$

where $q_{t-1}(\boldsymbol{\eta}):=\sum_{j=1}^{\infty} \exp \left(i \mathbf{z}^{\prime} \mathbf{Z}_{t-j}\right) \Psi_{j}(\omega)$.

Let us consider the NKPC written in a regression model (8). Notice that $h_{\varepsilon}(\boldsymbol{\eta}):=$ $E\left[\varepsilon_{t} q_{t-1}(\boldsymbol{\eta})\right]=0$, because $E\left[\varepsilon_{t} \mid \mathcal{F}_{t-1}\right]=0$ and $q_{t-1}(\boldsymbol{\eta})$ is $\mathcal{F}_{t-1}$-measurable. Then, substituting 
$\pi_{t}$ by $(4)$ in $h_{\pi}$, we obtain

$$
h_{\pi}^{c}(\boldsymbol{\eta})=h_{X}^{c}(\boldsymbol{\eta}) \boldsymbol{\theta}_{0}
$$

where $h_{X}(\boldsymbol{\eta}):=E\left[\mathbf{X}_{t} q_{t-1}(\boldsymbol{\eta})\right]$, with $\mathbf{X}_{t}=\left(1, \pi_{t-1}, \pi_{t+1}, x_{t}\right)^{\prime}$. In obtaining (21), we use the key equivalence between $h_{X}(\boldsymbol{\eta})$ and $E\left[\mathbf{X}_{t}^{e} q_{t-1}(\boldsymbol{\eta})\right]$, which follows from the law of iterated expectations. While the primitive integrated moment $E\left[\mathbf{X}_{t}^{e} q_{t-1}(\boldsymbol{\eta})\right]$ includes unobservables in $\mathbf{X}_{t}^{e}$, our integrated approach allows us to express the linear transformation in terms of observables $\mathbf{X}_{t}$. Let $\boldsymbol{\Xi}:=\left(\Lambda, \chi^{\prime}\right)^{\prime}$ be a random vector with independent components, $\Lambda$ uniformly distributed in $[0,1]$ and $\chi$ a standard Gaussian random vector in $\mathbb{R}^{d}$. Then, premultiplying $h_{X}(\boldsymbol{\eta})$ to both sides in (21), evaluating at $\boldsymbol{\eta}=\boldsymbol{\Xi}$, and taking expectations with respect to $\boldsymbol{\Xi}$ yields

$$
E\left[h_{X}(\boldsymbol{\Xi}) h_{\pi}^{c}(\boldsymbol{\Xi})\right]=E\left[h_{X}(\boldsymbol{\Xi}) h_{X}^{c}(\boldsymbol{\Xi})\right] \boldsymbol{\theta}_{0} .
$$

Notice that both $E\left[h_{X}(\boldsymbol{\Xi}) h_{X}^{c}(\boldsymbol{\Xi})\right]$ and $E\left[h_{X}(\boldsymbol{\Xi}) h_{\pi}^{c}(\boldsymbol{\Xi})\right]$ are real-valued and exist under the finite second moment conditions:

Assumption 1. (a) $E\left[\left|\mathbf{X}_{t}\right|^{2}\right]<\infty$ and $E\left[\left|\pi_{t}\right|^{2}\right]<\infty$; (b) The matrix $E\left[h_{X}(\boldsymbol{\Xi}) h_{X}^{c}(\boldsymbol{\Xi})\right]$ is non-singular; (c) $\left\{\mathbf{X}_{t}, \mathbf{Z}_{t}\right\}$ is a strictly stationary and ergodic time series vector.

From (22), and under Assumption 1(b) we conclude that $\boldsymbol{\theta}_{0}$ is identified.

These arguments suggest the GBSE, obtained as

$$
\widehat{\boldsymbol{\theta}}_{G B S E}=\left(E\left[\widehat{h}_{X}(\boldsymbol{\Xi}) \widehat{h}_{X}^{c}(\boldsymbol{\Xi})\right]\right)^{-1}\left(E\left[\widehat{h}_{X}(\boldsymbol{\Xi}) \widehat{h}_{\pi}^{c}(\boldsymbol{\Xi})\right]\right)
$$

where $\widehat{h}_{X}(\boldsymbol{\eta})=n^{-1} \sum_{t=1}^{n} \mathbf{X}_{t} \widehat{q}_{t-1}(\boldsymbol{\eta})$ with $\widehat{q}_{t-1}(\boldsymbol{\eta})=\sum_{j=1}^{t}\left(n / n_{j}\right)^{1 / 2} \exp \left(i \mathbf{z}^{\prime} \mathbf{Z}_{t-j}\right) \Psi_{j}(\omega)$ and $\widehat{h}_{\pi}(\boldsymbol{\eta})=n^{-1} \sum_{t=1}^{n} \pi_{t} \widehat{q}_{t-1}(\boldsymbol{\eta})$. Notice that $\left(n_{j} / n\right)^{1 / 2}$ is a finite-sample correction factor, making the finite-sample performance more precise by downweighting distant lags. Simple algebra shows that (23) is equivalent to (14).

To establish the asymptotic distribution of the proposed GBSE, we employ a Hilbert space approach. Let us consider the Hilbert space $L_{2}\left(\mathbb{R}^{d}, \nu\right)$ of all complex-valued and square $\nu$-integrable functions on $\mathbb{R}^{d}$, where $\nu$ is the product measure of the $\phi$-measure and the Lebesgue measure on $[0,1]$. The inner product is defined in $L_{2}\left(\mathbb{R}^{d}, \nu\right)$ as

$$
\langle f, g\rangle=\int_{\mathbb{R}^{d}} f(\boldsymbol{\eta}) g^{c}(\boldsymbol{\eta}) d \nu(\boldsymbol{\eta})=\int_{\mathbb{R}^{d}} f(\omega, \mathbf{z}) g^{c}(\omega, \mathbf{z}) \phi(d \mathbf{z}) d \omega,
$$

where $g^{c}$ denotes the complex conjugate of $g . L_{2}\left(\mathbb{R}^{d}, \nu\right)$ is endowed with the natural Borel $\sigma$-field induced by the norm $\|\cdot\|=\langle\cdot, \cdot\rangle^{1 / 2}$. We are now ready to prove Theorem 1 .

Proof of Theorem 1: Using the definition of the inner product in $L_{2}$, the GBSE can be 
expressed as

$$
\widehat{\boldsymbol{\theta}}_{G B S E}=\boldsymbol{\theta}_{0}+\left\langle\widehat{h}_{X}, \widehat{h}_{X}\right\rangle^{-1}\left\langle\widehat{h}_{X}, \widehat{h}_{\varepsilon}\right\rangle .
$$

Recall that $\widehat{h}_{X}(\boldsymbol{\eta})=n^{-1} \sum_{t=1}^{n} \mathbf{X}_{t} \widehat{q}_{t-1}(\boldsymbol{\eta})$ with $\widehat{q}_{t-1}(\boldsymbol{\eta})=\sum_{j=1}^{t}\left(n / n_{j}\right)^{1 / 2} \exp \left(i \mathbf{z}^{\prime} \mathbf{Z}_{t-j}\right) \Psi_{j}(\omega)$ and $\widehat{h}_{\varepsilon}(\boldsymbol{\eta})=n^{-1} \sum_{t=1}^{n} \varepsilon_{t} \widehat{q}_{t-1}(\boldsymbol{\eta})$.

Next, we show that a law of large numbers holds for $\widehat{h}_{X}$ in $L_{2}\left(\mathbb{R}^{d}, \nu\right)$. Write

$$
\begin{aligned}
\widehat{h}_{X}(\boldsymbol{\eta}) & =n^{-1} \sum_{t=1}^{n} \mathbf{X}_{t}\left(\sum_{j=1}^{t}\left(n / n_{j}\right)^{1 / 2} \exp \left(i \mathbf{z}^{\prime} \mathbf{Z}_{t-j}\right) \Psi_{j}(\omega)\right) \\
& =\sum_{j=1}^{n}\left(n / n_{j}\right)^{1 / 2}\left(n^{-1} \sum_{t=1}^{n} \mathbf{X}_{t} \exp \left(i \mathbf{z}^{\prime} \mathbf{Z}_{t-j}\right)\right) \Psi_{j}(\omega) .
\end{aligned}
$$

Define $\hat{\delta}_{j}(\mathbf{z})=n^{-1} \sum_{t=1}^{n} \mathbf{X}_{t} \exp \left(i \mathbf{z}^{\prime} \mathbf{Z}_{t-j}\right), \delta_{j}(\mathbf{z})=E\left[\mathbf{X}_{t} \exp \left(i \mathbf{z}^{\prime} \mathbf{Z}_{t-j}\right)\right]$ and

$$
h_{j, n}(\mathbf{z})=\left(n / n_{j}\right)^{1 / 2} \hat{\delta}_{j}(\mathbf{z})-\delta_{j}(\mathbf{z})
$$

so that, by the triangle inequality,

$$
\begin{aligned}
\left\|\widehat{h}_{X}-h_{X}\right\|^{2} & \leq\left\|\sum_{j=1}^{n} h_{j, n}(\cdot) \Psi_{j}(\cdot)\right\|^{2}+\left\|\sum_{j=1+n}^{\infty} \delta_{j}(\cdot) \Psi_{j}(\cdot)\right\|^{2} \\
& =o_{P}(1)+\sum_{j=1+n}^{\infty} \frac{\left\|\delta_{j}(\cdot)\right\|^{2}}{(j \pi)^{2}} \\
& =o_{P}(1),
\end{aligned}
$$

where the first equality follows from Lemma 1 in Escanciano and Velasco (2006), and the second from $\left\|\delta_{j}(\cdot)\right\|^{2} \leq E\left[\left|\mathbf{X}_{t}\right|^{2}\right]$ ( since $\left.\left|\exp \left(i \mathbf{z}^{\prime} \mathbf{Z}_{t-j}\right)\right| \leq 1\right)$.

The law of large numbers and the continuity of the inner product yield

$$
\sqrt{n}\left(\widehat{\boldsymbol{\theta}}_{G B S E}-\boldsymbol{\theta}_{0}\right)=\left\langle h_{X}, h_{X}\right\rangle^{-1} \sqrt{n}\left\langle h_{X}, \widehat{h}_{\varepsilon}\right\rangle+o_{P}(1)
$$

Furthermore, it can be shown that

$$
\sqrt{n}\left\langle h_{X}, \widehat{h}_{\varepsilon}\right\rangle=\frac{1}{\sqrt{n}} \sum_{t=1}^{n} \varepsilon_{t} H_{t-1},
$$

where $H_{t-1}:=\left\langle h_{X}, \widehat{q}_{t-1}\right\rangle=\left\langle E\left[\mathbf{X}_{t} q_{t-1}(\boldsymbol{\eta})\right], \widehat{q}_{t-1}\right\rangle$. Therefore, the theorem follows from the standard central limit theorem for strictly stationary and ergodic martingale difference se- 
quences.

\subsection{Bootstrap-based tests for identification}

To construct test statistics for (9) and (10), we modify the generalized spectrum test proposed in Escanciano (2006). Unlike the bootstrap procedure proposed in Escanciano (2006), the modified bootstrap-based test proposed here does not require reestimation of parameters in each bootstrap iteration, which leads to considerable reduction in computational time.

With some abuse of notation, we denote $\varepsilon_{1 t}(\boldsymbol{\beta}):=\beta_{0}+\beta_{1} \pi_{t+1}+\left(1-\beta_{1}\right) x_{t}-\beta_{2} \pi_{t-1}$ and $\varepsilon_{2 t}(\boldsymbol{\beta}):=\pi_{t+1}-x_{t}-\beta_{3}-\beta_{4} \pi_{t-1}$, where $\boldsymbol{\beta}:=\left(\beta_{0}, \beta_{1}, \beta_{2}, \beta_{3}, \beta_{4}\right)^{\prime} \in \mathbb{R}^{5}$, and construct a test for the conditional moment restrictions, for $h=1$ and 2 ,

$$
E\left[\varepsilon_{h t}(\boldsymbol{\beta}) \mid I_{t-1}\right]=0 \text { a.s. for some } \boldsymbol{\beta} \in \mathbb{R}^{5}
$$

The parameters $\beta_{3}$ and $\beta_{4}$ can be estimated by Least Squares, whereas $\left(\beta_{0}, \beta_{1}, \beta_{2}\right)$ can be estimated by the GBSE. ${ }^{9}$

Assume that $\sigma\left(\mathbf{Z}_{t}, \mathbf{Z}_{t-1}, \cdots\right)$ is in the information set $I_{t}$, where $\mathbf{Z}_{t}$ is the selected vector of instruments. To deal with the infinite-dimensional information set in a feasible way, we follow the pairwise approach of Hong (1999) and Escanciano (2006), and test the infinite number of conditional moment restrictions

$$
E\left[\varepsilon_{h t}(\boldsymbol{\beta}) \mid \mathbf{Z}_{t-j}\right]=0 \text { for all } j \geq 1
$$

By well-known results, see Bierens (1990), we can characterize (25) by the following equation

$$
\gamma_{h j}(\mathbf{z}):=E\left[\varepsilon_{h t}(\boldsymbol{\beta}) \exp \left(i \mathbf{z}^{\prime} \mathbf{Z}_{t-j}\right)\right]=0, \text { for all } \mathbf{z} \in \mathbb{R}^{d}, \text { and all } j \geq 1
$$

where $i=\sqrt{-1}$ and $d$ is the dimension of $\mathbf{Z}_{t-j}$.

To test for the significance of all functions $\left\{\gamma_{h j}(\cdot)\right\}$ in (26), we extend the original idea in Escanciano (2006) to construct a test based on an integrated Fourier transform of the measures $\left\{\gamma_{h j}(\cdot)\right\}_{j=1}^{\infty}$. Notice that the sample analogue of $\gamma_{h j}(\cdot)$ is given by

$$
\widehat{\gamma}_{h j}(\mathbf{z}):=\frac{1}{n_{j}} \sum_{t=j}^{n} \widehat{\varepsilon}_{h t} \widehat{w}_{h, t-j}(\mathbf{z}), \quad j \geq 1
$$

where $n_{j}:=n-j+1, \widehat{\varepsilon}_{h t}:=\varepsilon_{h t}(\widehat{\boldsymbol{\beta}})$, with $\widehat{\boldsymbol{\beta}}$ denoting the OLS and GBSE estimates of $\boldsymbol{\beta}$,

\footnotetext{
${ }^{9}$ Notice that the use of GMM for (9) can lead to weak identification and hence misleading inferences.
} 
$\tilde{\pi}_{t-1}=\left(1, \pi_{t-1}\right)^{\prime}$, and

$$
\widehat{w}_{1, t-j}(\mathbf{z}):=\exp \left(i \mathbf{z}^{\prime} \mathbf{Z}_{t-j}\right)-\left(\sum_{t=j}^{n} \tilde{\pi}_{t-1}^{\prime} \exp \left(i \mathbf{z}^{\prime} \mathbf{Z}_{t-j}\right)\right)\left(\sum_{t=j}^{n} \tilde{\pi}_{t-1} \tilde{\pi}_{t-1}^{\prime}\right)^{-1} \tilde{\pi}_{t-1}
$$

and

$$
\widehat{w}_{2, t-j}(\mathbf{z}):=\exp \left(i \mathbf{z}^{\prime} \mathbf{Z}_{t-j}\right)-\left(\sum_{t=1}^{n} \mathbf{s}_{t} \mathbf{s}_{t}^{\prime}\right)^{-1}\left(\sum_{t=j}^{n} \mathbf{s}_{t} \exp \left(i \mathbf{z}^{\prime} \mathbf{Z}_{t-j}\right)\right) \mathbf{s}_{t},
$$

where $\mathbf{s}_{t}:=\left(1, \pi_{t+1}-x_{t}, \pi_{t-1}\right)^{\prime}$.

To aggregate all the information in $\left\{\widehat{\gamma}_{h j}(\cdot)\right\}_{j=1}^{n}$ we consider the random function

$$
S_{h n}(\omega, \mathbf{z}):=\sum_{j=1}^{n} n_{j}^{1 / 2} \widehat{\gamma}_{h j}(\mathbf{z}) \frac{\sqrt{2} \sin (j \pi \omega)}{j \pi}
$$

The null will be rejected when $S_{h n}$ takes "large" absolute values. Our test statistics is the Cramér-von Mises $(C v M)$ type statistic

$$
C v M_{h n}:=\int_{\mathbb{R}^{d} \times[0,1]}\left|S_{h n}(\omega, \mathbf{z})\right|^{2} \phi(\mathbf{z}) d \mathbf{z} d \omega
$$

where $\phi$ is the standard Gaussian density. After some algebra, we can obtain a quadratic matrix form for our test statistic, which can be efficiently implemented as

$$
C v M_{h n}=\sum_{j=1}^{n} \frac{n_{j}}{(j \pi)^{2}} \int_{\mathbb{R}^{d}}\left|\widehat{\gamma}_{h j}(\mathbf{z})\right|^{2} \phi(\mathbf{z}) d \mathbf{z}
$$

Using the results in Escanciano (2006), one can easily show that $\mathbf{S}_{n}:=\left(S_{1 n}, S_{2 n}\right)$ converges weakly as a stochastic process in a suitable Hilbert space. The asymptotic distribution of $C v M_{h n}$ will follow from an application of the continuous mapping theorem. This asymptotic distribution cannot be tabulated in general, and depends in a non-trivial manner on the true data generating process. To overcome this problem, we suggest to implement the test with the assistance of a simple boostrap procedure. An alternative test that does not require bootstrap is proposed in Chen, Choi, and Escanciano (2017).

We consider the bootstrap analogue of $\widehat{\gamma}_{h j}(\mathbf{z})$ as

$$
\widehat{\gamma}_{h j}^{*}(\mathbf{z}):=\frac{1}{n_{j}} \sum_{t=j}^{n} V_{t} \widehat{\varepsilon}_{h t} \widehat{w}_{h, t-j}(\mathbf{z}), \quad j \geq 1
$$

where $\left\{V_{t}\right\}_{t=1}^{n}$ is a sequence of i.i.d. random variables with zero mean, unit variance and 
bounded support and that are independent of the original data.

To generate the bootstrap analogue $\widehat{\gamma}_{h j}^{*}(x)$, we employ a sequence $\left\{V_{t}\right\}$ of i.i.d. Bernoulli variates with $P\left(V_{t}=0.5(1-\sqrt{5})\right)=b$ and $P\left(V_{t}=0.5(1+\sqrt{5})\right)=1-b$ where $b=$ $(1+\sqrt{5}) / 2 \sqrt{5}$, as suggested in e.g., Mammen (1993). Notice that $\widehat{w}_{h, t-j}$ can be interpreted as a least squares residual from a regression of the weight $\exp \left(i \mathbf{z}^{\prime} \mathbf{Z}_{t-j}\right)$ into the score $\mathbf{s}_{t}$. We then use $\left\{\widehat{\gamma}_{h j}^{*}(\cdot)\right\}_{j=1}^{n}$ and compute

$$
C v M_{h n}^{*}:=\sum_{j=1}^{n} \frac{n_{j}}{(j \pi)^{2}} \int_{\mathbb{R}^{d}}\left|\widehat{\gamma}_{h j}^{*}(\mathbf{z})\right|^{2} \phi(\mathbf{z}) d \mathbf{z} .
$$

Independent replications can be used to approximate the bootstrap critical value of $C v M_{h n}^{*}$ at $\tau$-th level as accurately as desired. Let denote by $c_{h n, \tau}^{*}$ such bootstrap approximation of the critical value. Our bootstrap test rejects the null of lack of identification if $C v M_{h n}>c_{h n, \tau}^{*}$, for $h=1,2$. The consistency of the proposed bootstrap can be established using the same arguments as in Escanciano (2006), and hence its proof is omitted here. 


\section{$\begin{array}{ll}7 & \text { Tables and Figures }\end{array}$}

Table 1: Regression Model 1

\begin{tabular}{ccccc}
\hline \hline & $\delta_{0}$ & $\delta_{1}$ & $\delta_{2}$ & $\delta_{3}$ \\
\hline Coefficient & -0.3683 & -3.0569 & -4.0952 & 2.8137 \\
$t$-statistic & -0.7959 & -5.9750 & -2.6883 & 3.5579 \\
$F$-test & $H_{0}: \delta_{2}=\delta_{3}=0$ & $p$-value: & 0.000 \\
\hline
\end{tabular}

Table 2: Regression Model 2

\begin{tabular}{cccccc}
\hline \hline & $\alpha_{0}$ & $\alpha_{1}$ & $\alpha_{2}$ & $\alpha_{3}$ & $\alpha_{4}$ \\
\hline Coefficient & 0.1332 & -0.9684 & 0.6908 & 1.6363 & -0.6552 \\
$t$-statistic & 2.6837 & -95.6595 & 8.5814 & 2.2095 & -1.9922 \\
$F$-test & $H_{0}: \alpha_{3}=\alpha_{4}=0$ & \multicolumn{4}{c}{$p$-value: 0.000} \\
\hline
\end{tabular}

Table 3: Estimation for the US NKPC, 1970Q1-1998Q2

\begin{tabular}{ccccccccc}
\hline \hline & \multicolumn{4}{c}{ GMM(Mavroeidis et al. (2014)) } & \multicolumn{4}{c}{ GBSE Estimator at High-frequency } \\
\hline \multirow{2}{*}{ Vintage } & $\gamma_{0}$ & $\hat{\gamma_{f}}$ & $\hat{\gamma_{b}}$ & $\hat{\lambda}$ & $\gamma_{0}$ & $\hat{\gamma_{f}}$ & $\hat{\gamma_{b}}$ & $\hat{\lambda}$ \\
\hline \multirow{2}{*}{1998} & 0.041 & 0.615 & 0.340 & 0.026 & 0.089 & 0.466 & 0.442 & 0.031 \\
& $(0.030)$ & $(0.057)$ & $(0.058)$ & $(0.013)$ & $(0.044)$ & $(0.029)$ & $(0.034)$ & $(0.015)$ \\
\hline \multirow{2}{*}{2012} & -0.049 & 0.719 & 0.240 & 0.018 & -0.011 & 0.448 & 0.430 & 0.025 \\
& $(0.040)$ & $(0.099)$ & $(0.095)$ & $(0.012)$ & $(0.028)$ & $(0.032)$ & $(0.045)$ & $(0.011)$ \\
\hline
\end{tabular}

Note: We use four lags of inflation and two lags of the labor share, wage inflation, and quadratically-detrended output as instruments. 
Table 4: Robustness Check 1: GBSE with Different Instrument Variables

\begin{tabular}{ccccccccc}
\hline \hline & \multicolumn{3}{c}{ Vintage Data in 1998 } & \multicolumn{4}{c}{ Vintage Data in 2012} \\
\hline & $\gamma_{0}$ & $\hat{\gamma_{f}}$ & $\hat{\gamma}_{b}$ & $\hat{\lambda}$ & $\gamma_{0}$ & $\hat{\gamma_{f}}$ & $\hat{\gamma}_{b}$ & $\hat{\lambda}$ \\
& $($ s.e. $)$ & $($ s.e. $)$ & $($ s.e. $)$ & $($ s.e. $)$ & $($ s.e. $)$ & $($ s.e. $)$ & $($ s.e. $)$ & $($ s.e. $)$ \\
\hline Bench- & 0.089 & 0.466 & 0.442 & 0.031 & -0.011 & 0.448 & 0.430 & 0.025 \\
mark & $(0.044)$ & $(0.029)$ & $(0.034)$ & $(0.015)$ & $(0.027)$ & $(0.032)$ & $(0.045)$ & $(0.011)$ \\
\hline Z1 & 0.185 & 0.413 & 0.406 & 0.035 & -0.011 & 0.419 & 0.405 & 0.037 \\
& $(0.048)$ & $(0.042)$ & $(0.045)$ & $(0.016)$ & $(0.040)$ & $(0.062)$ & $(0.066)$ & $(0.013)$ \\
\hline Z2 & 0.067 & 0.492 & 0.432 & 0.024 & -0.015 & 0.468 & 0.438 & 0.021 \\
& $(0.032)$ & $(0.028)$ & $(0.031)$ & $(0.012)$ & $(0.027)$ & $(0.032)$ & $(0.045)$ & $(0.011)$ \\
\hline
\end{tabular}

Note: Z1includes 4 lags of inflation, labor share, output gap, yield spread, wage inflation and commodity inflation. Z2 includes 4 lags of inflation and 3 lags of labor share.

Table 5: Robustness Check 2: GBSE with Different Specifications of Forcing Variable

\begin{tabular}{ccccccccc}
\hline \hline & \multicolumn{4}{c}{ Vintage Data in 1998} & \multicolumn{3}{c}{ Vintage Data in 2012} \\
\hline & $\gamma_{0}$ & $\hat{\gamma}_{f}$ & $\hat{\gamma}_{b}$ & $\hat{\lambda}$ & $\gamma_{0}$ & $\hat{\gamma_{f}}$ & $\hat{\gamma}_{b}$ & $\hat{\lambda}$ \\
Forcing Variables & $($ s.e. $)$ & $($ s.e. $)$ & $($ s.e. $)$ & $($ s.e. $)$ & $($ s.e. $)$ & $($ s.e. $)$ & $($ s.e. $)$ & $($ s.e. $)$ \\
\hline Bench- & 0.089 & 0.466 & 0.442 & 0.031 & -0.011 & 0.448 & 0.430 & 0.025 \\
mark & $(0.044)$ & $(0.029)$ & $(0.034)$ & $(0.015)$ & $(0.024)$ & $(0.045)$ & $(0.048)$ & $(0.008)$ \\
\hline $\mathrm{X} 1$ & -0.005 & 0.500 & 0.458 & 0.026 & -0.006 & 0.526 & 0.409 & 0.018 \\
& $(0.043)$ & $(0.028)$ & $(0.037)$ & $(0.012)$ & $(0.017)$ & $(0.041)$ & $(0.047)$ & $(0.007)$ \\
\hline $\mathrm{X} 2$ & 0.037 & 0.489 & 0.432 & 0.020 & -0.000 & 0.504 & 0.413 & 0.023 \\
& $(0.033)$ & $(0.026)$ & $(0.030)$ & $(0.012)$ & $(0.024)$ & $(0.049)$ & $(0.051)$ & $(0.009)$ \\
\hline
\end{tabular}

Note: The specification of X1 is based on Nason and Smith (2008a) while X2 is based on Sbordone (2005). 
Table 6: Robustness Check 3: Different Dependent Variables

\begin{tabular}{ccccccccc}
\hline \hline & \multicolumn{3}{c}{ Vintage Data in 1998 } & \multicolumn{4}{c}{ Vintage Data in 2012} \\
\hline & $\gamma_{0}$ & $\hat{\gamma_{f}}$ & $\hat{\gamma_{b}}$ & $\hat{\lambda}$ & $\gamma_{0}$ & $\hat{\gamma_{f}}$ & $\hat{\gamma_{b}}$ & $\hat{\lambda}$ \\
& $($ s.e. $)$ & $($ s.e. $)$ & $($ s.e. $)$ & $($ s.e. $)$ & $($ s.e. $)$ & $($ s.e. $)$ & $($ s.e. $)$ & $($ s.e. $)$ \\
\hline Bench- & 0.089 & 0.466 & 0.442 & 0.031 & -0.011 & 0.448 & 0.430 & 0.025 \\
mark & $(0.044)$ & $(0.029)$ & $(0.034)$ & $(0.015)$ & $(0.027)$ & $(0.032)$ & $(0.045)$ & $(0.011)$ \\
\hline CPI & 0.245 & 0.405 & 0.357 & 0.062 & 0.135 & 0.367 & 0.359 & 0.035 \\
& $(0.093)$ & $(0.067)$ & $(0.082)$ & $(0.029)$ & $(0.053)$ & $(0.072)$ & $(0.080)$ & $(0.013)$ \\
\hline \multirow{2}{*}{ PCE } & 0.084 & 0.489 & 0.432 & 0.020 & -0.005 & 0.447 & 0.446 & 0.022 \\
& $(0.037)$ & $(0.030)$ & $(0.037)$ & $(0.010)$ & $(0.026)$ & $(0.029)$ & $(0.026)$ & $(0.009)$ \\
\hline
\end{tabular}

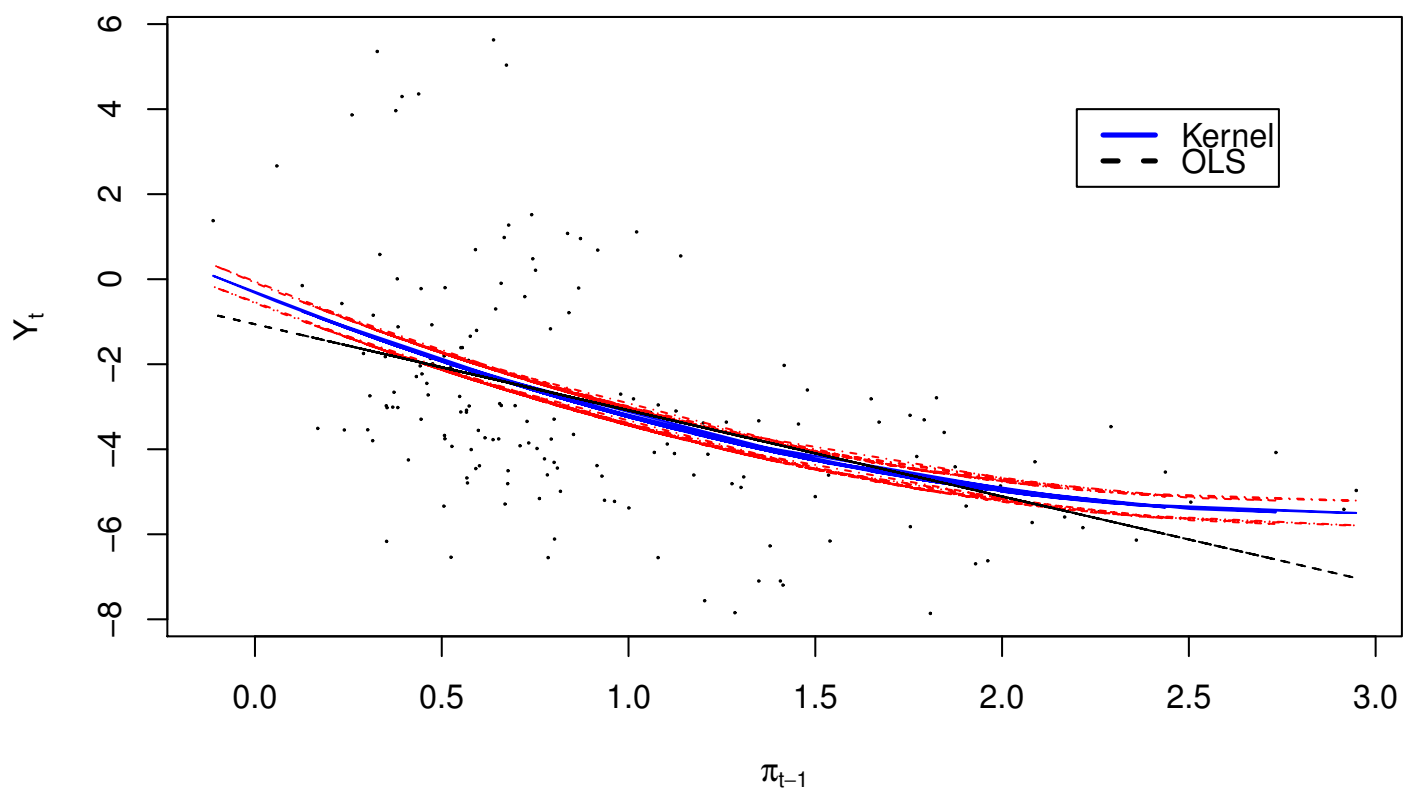

Figure 1: The Relation between $Y_{t+1}$ and $\pi_{t-1}$ 


\section{References}

Assenmacher-Wesche, K. and S. Gerlach (2008a): "Interpreting euro area inflation at high and low frequencies," European Economic Review, 52, 964-986.

(2008b): "Money growth, output gaps and inflation at low and high frequency: Spectral estimates for Switzerland," Journal of Economic Dynamics and Control, 32, 411435.

Baxter, M. And R. G. King (1999): "Measuring Business Cycles: Approximate BandPass Filters For Economic Time Series," The Review of Economics and Statistics, 81, $575-593$.

Berkowitz, J. (2001): "Generalized spectral estimation of the consumption-based asset pricing model," Journal of Econometrics, 104, 269-288.

Bierens, H. (1990): "A consistent conditional moment test of functional form," Econometrica, 58, 1443-1458.

Calvo, G. A. (1983): "Staggered Prices in a Utility-Maximizing Framework," Journal of Monetary Economics, 12, 383-398.

Chen, B., J. Choi, And J. C. Escanciano (2017): "Testing for Fundamental Vector Moving Average Representations," Quantitative Economics, 8, 149-180.

Cogley, T. And A. Sbordone (2008): "Trend Inflation, Indexation, and Inflation Persistence in the New Keynesian Phillips Curve," American Economic Review, 98, 2101-26.

Davig, T. And E. M. Leeper (2007): "Generalizing the Taylor principle," The American economic review, 97, 607-635.

(2008): "Endogenous monetary policy regime change," in NBER International Seminar on Macroeconomics 2006, University of Chicago Press, 345-391.

Dolado, J., R. M.-D. Pedrero, and F. J. Ruge-Murcia (2004): "Nonlinear monetary policy rules: some new evidence for the US," Studies in Nonlinear Dynamics 8 Econometrics, 8.

Dominguez, M. A. And I. N. Lobato (2004): "Consistent estimation of models defined by conditional moment restrictions," Econometrica, 72, 1601-1615. 
Dufour, J.-M., L. Khalaf, And M. Kichian (2006): "Inflation dynamics and the New Keynesian Phillips Curve: An Identification Robust Econometric Analysis," Journal of Economic Dynamics and Control, 30, 1707-1727.

Engle, R. F. (1974): "Band Spectrum Regression," International Economic Review, 15, $1-11$.

Escanciano, J. C. (2006): "Goodness-of-Fit Tests for Linear and Nonlinear Time Series Models," Journal of the American Statistical Association, 101, 531-541.

(2017): "A simple and robust estimator for linear regression models with strictly exogenous instruments," The Econometrics Journal.

Escanciano, J. C. And S. Mayoral (2010): "Data-driven smooth tests for the martingale difference hypothesis," Computational Statistics and Data Analysis, 54, 1983-1998.

Escanciano, J. C. And C. Velasco (2006): "Generalized spectral tests for the martingale difference hypothesis," Journal of Econometrics, 134, 151-185.

Fuhrer, J. AND G. MoOre (1995): "Inflation persistence," The Quarterly Journal of Economics, 110, 127-159.

Galí, J. And M. Gertler (1999): "Inflation Dynamics: A Structural Econometric Analysis," Journal of Monetary Economics, 44, 195-222.

Gali, J., M. Gertler, And J. D. Lopez-Salido (2001): "European Inflation Dynamics," European Economic Review, 45, 1237-1270.

Hannan, E. (1967): "Canonical correlation and multiple equation systems in economics," Econometrica, 35, 123-138.

Hannan, E. J. (1965): “The estimation of relationships involving distributed lags," Econometrica, 33, 206-224.

Hansen, L. P. (1982): "Large Sample Properties of Generalized Method of Moments Estimators," Econometrica, 50, 1029-54.

Hansen, L. P., J. Heaton, And A. Yaron (1996): "Finite-sample properties of some alternative GMM estimators," Journal of Business 8 Economic Statistics, 14, 262-280.

Hong, Y. (1999): "Hypothesis Testing in Time Series via the Empirical Characteristic Function: A Generalized Spectral Density Approach," Journal of the American Statistical Association, 94, 1201-1220. 
Kleibergen, F. (2002): "Pivotal Statistics for Testing Structural Parameters in Instrumental Variables Regression," Econometrica, 70, 1781-1803.

Kleibergen, F. And S. Mavroeidis (2009): "Weak Instrument Robust Tests in GMM and the New Keynesian Phillips Curve," Journal of Business 86 Economic Statistics, 27, $293-311$.

MA, A. (2002): "GMM Estimation of the New Phillips Curve," Economics Letters, 76, 411-417.

Mammen, E. (1993): "Bootstrap and wild bootstrap for high dimensional linear models," The annals of statistics, 255-285.

Mavroeidis, S. (2005): "Identification Issues in Forward-Looking Models Estimated by GMM, with an Application to the Phillips Curve," Journal of Money, Credit and Banking, $37,421-48$.

(2010): "Monetary policy rules and macroeconomic stability: some new evidence," The American Economic Review, 100, 491-503.

Mavroeidis, S., M. Plagborg-Møller, And J. H. Stock (2014): "Empirical evidence on inflation expectations in the New Keynesian Phillips Curve," Journal of Economic Literature, 52, 124-188.

Nason, J. M. And G. W. Smith (2008a): "Identifying the New Keynesian Phillips Curve," Journal of Applied Econometrics, 23, 525-551.

- (2008b): "The New Keynesian Phillips Curve: Lessons From Single-Equation Econometric Estimation," Federal Reserve Bank of Richmond Economic Quarterly, 361-395.

Orphanides, A. And D. W. Wilcox (2002): "The opportunistic approach to disinflation," International finance, 5, 47-71.

Phillips, A. W. (1958): "The relation between unemployment and the rate of change of money wage rates in the United Kingdom, 1861-1957," economica, 25, 283-299.

Roberts, J. M. (1995): "New Keynesian economics and the Phillips curve," Journal of money, credit and banking, 27, 975-984.

Rudd, J. AND K. Whelan (2007): "Modeling inflation dynamics: A critical review of recent research," Journal of Money, Credit and Banking, 39, 155-170. 
Sbordone, A. M. (2002): "Prices and unit labor costs: a new test of price stickiness," Journal of Monetary economics, 49, 265-292.

(2005): "Do expected future marginal costs drive inflation dynamics?" Journal of Monetary Economics, 52, 1183-1197.

Schmitt-Grohé, S. And M. Uribe (2008): "Policy implications of the New Keynesian Phillips curve," Federal Reserve Bank of Richmond Economic Quarterly.

Staiger, D. And J. H. Stock (1997): "Instrumental Variables Regression with Weak Instruments," Econometrica, 65, 557-586.

Stock, J. H., J. H. Wright, And M. Yogo (2002): "A Survey of Weak Instruments and Weak Identification in Generalized Method of Moments," Journal of Business \& Economic Statistics, 20, 518-29.

Stock, J. H. And M. Yogo (2005): "Testing for Weak Instruments in Linear IV Regression," in Identification and Inference for Econometric Models: Essays in Honor of Thomas Rothenberg, ed. by D. W. K. Andrews and J. H. Stock, Cambridge: Cambridge University Press, 80-108.

Wang, J. AND E. Zivot (1998): "Inference on Structural Parameters in Instrumental Variables Regression with Weak Instruments," Econometrica, 66, 1389-1404.

Zhu, F. (2005): "The Fragility of the Phillips Curve: A bumpy ride in the frequency domain," BIS working Paper, No.183. 